\title{
Modeling of transport in polycrystalline organic semiconductor films
}

\author{
S. Verlaak, ${ }^{\text {a),b) }}$ V. Arkhipov, and P. Heremans ${ }^{\text {a) }}$ \\ IMEC, Kapeldreef 75, B-3001 Leuven, Belgium
}

(Received 15 July 2002; accepted 5 December 2002)

\begin{abstract}
We propose a grain-boundary barrier model with an energy distribution of interfacial traps to describe charge transport in polycrystalline organic thin films. The model is applied to the interpretation of charge transport in unintentionally doped pentacene films. It gives an acceptable explanation for the concomitant increase in threshold voltage and mobility, and allows an understanding of the difference between the dopant-concentration and gate-voltage dependences of the mobility. (C) 2003 American Institute of Physics. [DOI: 10.1063/1.1541112]
\end{abstract}

Organic electronic devices promise a low-cost technology for large-area electronics. Among them, organic thinfilm transistors (OTFT's) based on polycrystalline pentacene have demonstrated promising performance. Pentacene was one of the first organic semiconductors to show relatively high room-temperature mobilities in thin films. ${ }^{1}$ Despite the fast technological evolution of OTFTs, the charge-transport mechanism in this material is still subject to debate. It was recently suggested that the concept of trap-controlled transport provides a more satisfactory description of carrier kinetics in polycrystalline pentacene OTFTs than a grainboundary barrier model, such as the Levinson model. ${ }^{2,3}$ However, the former assumes a spatially homogeneous distribution of traps, thereby disregarding the polycrystalline nature of those films, in which traps are known to be concentrated at grain boundaries. Moreover, the assumption of a trap distribution, rather than a monoenergetic trap state at the grain boundary as in the Levinson model, is more realistic in the case of organic semiconductors. ${ }^{4}$ In the present letter, we suggest an extended barrier model with an energy distribution of traps at grain boundaries. We apply this model to the analysis of charge transport in unintentionally doped polycrystalline thin films of pentacene. Experimentally verifiable distinctions between different models are indicated.

Within the amorphous phase separating two crystalline grains, a rather high density of localized states distributed within a broad energy range is expected. This distribution $g(E)$ will trap $\Sigma$ charges per unit interfacial area, as determined by the Fermi-Dirac statistics. Using the conventions in Fig. 1, drawn for hole conduction, $\Sigma$ can then be expressed as

$$
\Sigma=\int_{0}^{\infty} \frac{g(E)}{1+\exp \left(\frac{E_{B}+E_{F}\left(N_{A}\right)-E}{k T}\right)} d E,
$$

where $E_{F}$ is the Fermi level determined by the acceptorlike dopant concentration $N_{A}$ in crystalline grains, $k$ is the Boltzmann constant, and $T$ is the temperature. Those trapped charges are concentrated at grain boundaries and repel other charges of the same sign. For the film to remain electrically

${ }^{a}$ Also with: Department of Electrical Engineering, Katholieke Universiteit, Leuven, Belgium.

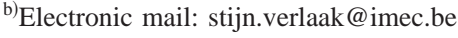

neutral, the trapped charges need to be screened by charges of the opposite sign. In doped films, ionized dopants provide the counter charges to screen the trapped charges at the grain boundary. The screening charges are located in a spacecharge region of the width $w$ (Fig. 1), and generate a potential barrier of the height $E_{B}$, as stated by the Poisson equation. ${ }^{5,6}$ The dopant concentration $N_{A}$ together with $\Sigma$ determines $w$ and $E_{B}$. In a transistor configuration, part of the screening charge is also located at the gate. In the latter case, the barrier height can only be calculated by solving the Poisson equation in two dimensions. We restrict the following calculations for the one-dimensional case, only including dopant concentration dependences. If the dopant concentration and, therefore, the density of ionized dopants are high enough, the width of the potential barrier will be smaller than the size of the grains, i.e., the grains will not be completely depleted. This is often the case in organic thin films for transistors. The Poisson equation then yields

$$
E_{B}=\frac{e^{2} \Sigma^{2}}{8 \varepsilon_{s} N_{A}}
$$

with $\varepsilon_{s}$ being the dielectric constant of the semiconductor and $e$ is the elementary charge. The mobility in a polycrystalline film is controlled by the rate of thermionic carrier jumps across the grain boundary (Fig. 1) as ${ }^{5}$

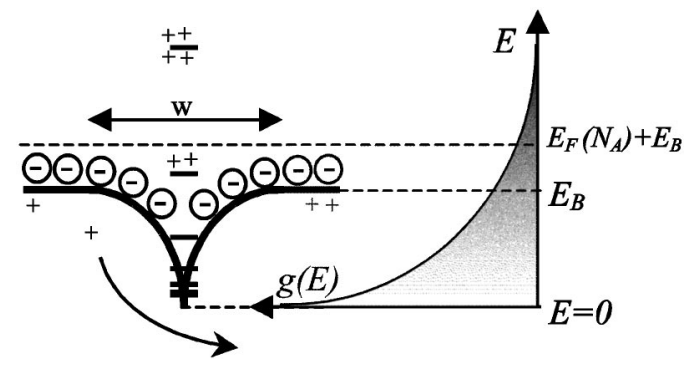

FIG. 1. Shown is the hole transport band of a polycrystalline semiconductor. Zero energy is at the highest point of the barrier for mathematical convenience. An interfacial hole-trap distribution at the boundary between two semi-infinite grains is partially filled with mobile holes coming from dopant molecules. The number of trapped holes depends on the trap distribution and the position of this distribution with respect to the Fermi level. This position is determined by the barrier height. The barrier width $w$ is the width over which dopants are negatively ionized in order to screen the trapped positive charges at the grain boundary. 


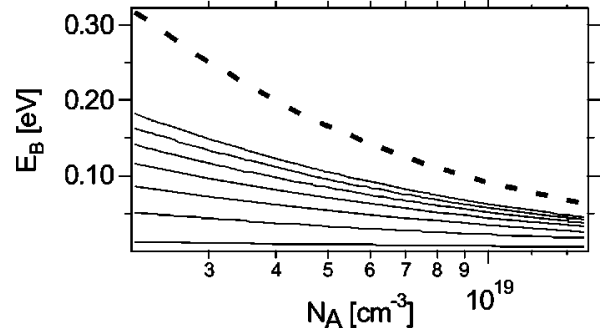

FIG. 2. Variation of the barrier height with dopant concentration $N_{A}$. An exponential trap distribution $g(E)=\Sigma_{0} / E_{0} \exp \left(-E / E_{0}\right)$ is assumed. Here $\Sigma_{0}$ is the total interfacial trap density, taken to be $5 \times 10^{12} \mathrm{~cm}^{-2}$, and $E_{0}$ is the characteristic energy of the distribution, varying from 0.1 (lowest curve) to $0.7 \mathrm{eV}$ (highest solid curve). The dashed line is modeled using $E_{0}$ $=8 \mathrm{eV}$, i.e., a uniform trap distribution.

$$
\mu \approx \mu_{0} \cdot \exp \left(-\frac{E_{B}}{k T}\right),
$$

with $\mu_{0}$ linearly increasing with grain size. Equation (2) shows that higher dopant concentrations screen the charges at the grain boundary more effectively and the barrier is lower. As illustrated in Fig. 1, this can be understood because a larger dopant concentration results in smaller space-charge regions at the grain barriers and, hence, lower barriers. From Eq. (2), it is also clear that the barrier height increases when more charges are trapped at the grain boundary. However, Eq. (1) and Fig. 1 show that higher potential barriers lift the trap distribution away from the Fermi level. Hence, increasing the potential barrier height self-consistently decreases the amount of charge trapped at the boundary. Solving Eqs. (1) and (2) for a given interfacial trap distribution at the grain boundaries results in self-consistent values of $\Sigma$ and $E_{B}$.

We simulated the dopant-concentration dependence of the barrier height for an exponential distribution of interfacial traps. The results are shown in Fig. 2. The major effect of varying the total trap density at the grain boundary is to scale the barrier height and, therefore, the mobility according to Eq. (3). Changing the steepness of the interfacial trap distribution on the other hand, modulates the variation of the barrier height with the dopant concentration. Barrier heights vary less with dopant concentration if the distribution is steeper. This feature distinguishes our model from the Levinson barrier model of a completely filled monoenergetic trap state. The latter necessarily results in the barrier height varying inversely proportionally to the dopant concentration. ${ }^{3}$

We have used our model to interpret experiments in which unintentional doping determines the barrier height and, thereby, the mobility. As-received pentacene (Aldrich, $98 \%$ pure) was grown in thin films with various deposition rates and substrate temperatures on thermally oxidized doped silicon wafers. Prior to growth, the $200 \mathrm{~nm}$ thick $\mathrm{SiO}_{2}$ was cleaned in oxygen plasma. The films grew with compact grains. Grain sizes varied between 300 and $800 \mathrm{~nm}$. For transistor measurements, gold source and drain contacts were evaporated on top of the pentacene film, while the wafer itself was used as a gate contact. For comparison, similar films and devices were made from pentacene purified once by vacuum gradient sublimation with a steep temperature gradient. The hole mobility $\mu$ and threshold voltage $V_{t}$ were extracted from the saturation regime. Large and positive

Downloaded 29 Jan 2003 to 146.103.254.11. Redistribution subject

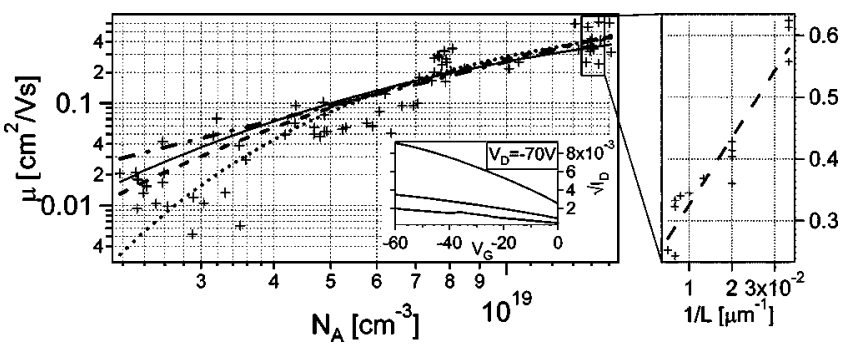

FIG. 3. Mobility vs dopant concentration in unintentionally doped polycrystalline as-received pentacene thin films. Data (crosses) is fitted assuming an exponential interfacial grain-boundary trap distribution (solid line) with $\Sigma_{0}$ $=4 \times 10^{12} \mathrm{~cm}^{-2}, E_{0}=0.5 \mathrm{eV}$, and $\mu_{0}$ assumed $1 \mathrm{~cm}^{2} / \mathrm{V} \mathrm{s}$. Also shown is a comparison with fits using the MTR model with exponential trap distribution (dashed-dotted), the Levinson model (dotted), and the potential well model (dashed line). The inset shows the transfer characteristics from which the mobilities and threshold voltages were extracted, for channel lengths of 30 (upper curve), 75, and $100 \mu \mathrm{m}$ (lowest curve) and a $2000 \mu \mathrm{m}$ channel width. For one specific thin film, mobilities of different devices with channel lengths $L=30,50,75,100,125,150$, and $200 \mu \mathrm{m}$ are plotted vs $L^{-1}$ on the right-hand side.

threshold voltages required large and negative drain voltages to keep the device in saturation. This requirement limited the range of gate voltages that could be applied, and devices could rarely be fully depleted. In practice, the mobility and threshold voltages were always extracted from the tangent to the transfer curve at zero-gate voltage. At this operating point, there are hardly any gate charges to screen the trapped charges at the grain boundaries and the maximum barrier height in the channel will predominantly be determined by the dopant concentration. Some transfer characteristics are shown in the inset to Fig. 3.

Depending upon film deposition conditions, the mobility varied from 0.005 to $0.6 \mathrm{~cm}^{2} / \mathrm{V} \mathrm{s}$ with a concomitant increase of the threshold voltage from less than +10 to about $+80 \mathrm{~V}$. Comparable mobilities and threshold voltages have been reported for as-received pentacene films before. ${ }^{1}$ In similar films of purified pentacene, the threshold voltage remained significantly lower and varied around $0 \mathrm{~V}$, independent of the mobility. These observations suggest that large values of the threshold voltage in films of as-received pentacene can be largely attributed to unintentional impurity doping, introducing acceptor states. The concentration of acceptor states (neutral when empty, negative when charged with an electron) able to generate mobile holes at room temperature in the as-received material, $N_{A}$, varied with the deposition conditions used. At high threshold voltages $V_{t}, N_{A}$ can be estimated as $N_{A}=\left(C_{i} V_{t}\right) /(e d)$. Here, $C_{i}$ is the gate capacitance per unit area, and $d$ is the effective film thickness, which was estimated to be about $5 \mathrm{~nm}$. Furthermore, the relatively small variation in grain size just mentioned, can, in first approximation, be disregarded because it cannot be responsible for the two orders of magnitude variation in mobility. The observed dependence of the mobility upon the dopant concentration is plotted in Fig. 3.

Based on the data in Fig. 3 alone, it is neither possible to clearly distinguish a grain-boundary barrier model from the multiple-trapping-and-release (MTR) model with an exponential bulk trap distribution, ${ }^{7}$ nor from the potential well model. ${ }^{8,9}$ This distinction is possible, however, when comparing the dopant-concentration dependence with the gatevoltage dependence of the mobility. The MTR model preto AIP license or copyright, see http://ojps.aip.org/aplo/aplcr.jsp 
dicts that the availability of more charges will result in filling more traps, thereby reducing the density of vacant traps and increasing the effective mobility of the remaining mobile charges. Similarly, the potential well model suggests that only mobile charges neutralize the counter charges of the trap states at the grain boundary. More mobile charges provide a better screening of those counter charges. In both of these models, the dopant-concentration dependence of the mobility is the same as its gate-voltage dependence: Changing the gate voltage or the dopant concentration is just a means to change the concentration of mobile charges in the semiconductor. For the barrier model, the situation is different. Changing the dopant concentration will not only change the number of mobile charges but also the size of the spacecharge region of ionized dopants that determines the potential barrier. Changing the gate voltage on the other hand, changes the number of mobile charges inside the semiconductor as well, but not the concentration of ionized dopants. Screening of the trapped charges at the grain boundary occurs partly by residual fixed ionized dopants in the semiconductor, and partly by the mobile counter charges at the gate. Contrary to ionized dopants inside the thin film, the counter charges at the gate are separated from the trapped charges by the gate dielectric. Hence, in a grain-boundary barrier model, the screening of the trapped charges by the gate is expected to have longer characteristic screening lengths, resulting in higher potential barriers. Therefore, for a given concentration of mobile charges, the mobility will be higher in films in which those mobile charges are generated by dopants.

This feature can be used to distinguish experimentally whether the mobility is limited by potential barriers at grain boundaries, trapping (MTR), or potential wells. In our pentacene films, we observed mobilities of around $0.1 \mathrm{~cm}^{2} / \mathrm{V} \mathrm{s}$ in films of as-received pentacene with a dopant-induced concentration of $5 \times 10^{12} \mathrm{~cm}^{-2}$ mobile charges in the first monolayers of the film. In a morphologically similar film ${ }^{10}$ of purified pentacene with an estimated accumulated gate charge of $C_{i}\left(V_{G}-V_{t}\right) / e=5 \times 10^{12} \mathrm{~cm}^{-2}$, the mobility was about one order of magnitude smaller. This observation is an indication for the validity of the grain-boundary barrier model. It should be mentioned that we have also grown films with purified pentacene that have a high mobility combined with threshold voltages around zero, yet those films have different morphologies, e.g., larger dendritic grains instead of compact grains.

A second experimental observation is the channel-length dependence of the mobility. We observed higher mobilities for shorter channel lengths. In terms of a grain-boundary barrier model, the potential between the source and drain drops over a smaller number of grain boundaries in shorter channels. The higher local electric field over the barriers is expected to result in a pronounced barrier lowering effect. ${ }^{7,11}$ In terms of a trapping model, transport becomes field depen- dent in strong electric fields as well. ${ }^{12}$ However, now, the potential drops uniformly across the channel instead of steeply across a limited number of grain-boundary barriers. The lower local fields associated with this uniform potential drop make this process less likely given the source-drain voltages applied to our transistors. The channel length effect is partly responsible for the data scatter in Fig. 3. To illustrate this, the right-hand side part of Fig. 3 shows the channellength dependence of the mobility measured on the same pentacene film.

In summary, we propose a model of charge transport in polycrystalline organic thin films controlled by carrier jumps across grain-boundary barriers with an interfacial trap distribution. This model proves to be more flexible than barrier models assuming monoenergetic trap states at the boundary and is, for most films, of practical importance for transistors physically more appropriate than multiple trapping and release of charge carriers. It should be noted however that for films with very low doping, small grains, and on thick gate dielectrics, the barriers can fully deplete the grains. ${ }^{5}$ In that case, the traps at the grain boundaries can safely be assumed to be homogeneously distributed in space, resulting in trapcontrolled transport. The model gives a reasonable explanation for the concomitant increase in the threshold voltage and the mobility in different films, and allows an understanding of the observed difference between the dopant-concentration and gate-voltage dependences of the mobility. Moreover, it suggests that some of the high mobilities reported in pentacene thin-film transistors might be caused by a high concentration of (unintentional) doping, rather than to a favorable thin-film morphology.

The authors gratefully acknowledge D. Janssen for conducting atomic force microscopy, B. Dutta for help with the vacuum equipment, and A. Verbist for the silicon wafers. One of the authors (S.V.) thanks IWT-Vlaanderen for financial support.

${ }^{1}$ Y. Y. Lin, D. J. Gundlach, S. F. Nelson, and T. N. Jackson, IEEE Trans. Electron Devices 44, 1325 (1997).

${ }^{2}$ R. A. Street, D. Knipp, and A. R. Völkel, Appl. Phys. Lett. 80, 1658 (2002).

${ }^{3}$ J. Levinson, F. R. Shepherd, P. J. Scanlon, W. D. Westwood, G. Este, and M. Rider, J. Appl. Phys. 53, 1193 (1982).

${ }^{4}$ E. A. Silinsh, Organic Molecular Crystals (Springer, Berlin, 1980), pp. 139-220.

${ }^{5}$ J. W. Orton and M. J. Powell, Rep. Prog. Phys. 43, 1265 (1980).

${ }^{6}$ G. Baccarani, B. Ricco, and G. Spadini, J. Appl. Phys. 49, 5565 (1978).

${ }^{7}$ G. Horowitz, M. E. Hajlaoui, and R. Hajlaoui, J. Appl. Phys. 87, 4456 (2000).

${ }^{8}$ J. H. Schön and B. Batlogg, Appl. Phys. Lett. 74, 260 (1999).

${ }^{9}$ H. Nussbaumer, F. P. Baumgartner, G. Willeke, and E. Bucher, J. Appl. Phys. 83, 292 (1998).

${ }^{10}$ Comparison between morphologies was made using atomic force microscopy.

${ }^{11}$ G. Horowitz and M. E. Hajlaoui, Adv. Mater. 12, 1046 (2000).

${ }^{12}$ S. E. Esipov, Phys. Rev. B 44, 7930 (1991). 